\title{
On the Capacity Region of the Gaussian MAC with Batteryless Energy Harvesting Transmitters
}

\author{
Omur Ozel Sennur Ulukus \\ Department of Electrical and Computer Engineering \\ University of Maryland College Park, MD 20742 \\ omur@umd.edu_ulukus@umd.edu
}

\begin{abstract}
We consider the two-user additive Gaussian multiple access channel (MAC) where the transmitters communicate by using energy harvested from nature. Energy arrivals of the users are i.i.d. in time, and for any given time, they are distributed according to a joint distribution. Energy arrivals cause timevariations for the amplitude constraints of the users. We first consider the static amplitude constrained Gaussian MAC and prove that the boundary of the capacity region is achieved by discrete input distributions of finite support. When both of the transmitters are equipped with no battery, Shannon strategies applied by users provide an inner bound for the capacity region. We prove that the boundary of this inner bound is achieved by input distributions with support set of zero Lebesgue measure.
\end{abstract}

\section{INTRODUCTION}

Energy harvesting capability provides sustainability and prolonged lifetime for wireless devices and this renders energy harvesting a desirable solution for many wireless networking applications. In such devices, energy arrives randomly from an exogenous energy source throughout the communication session and excess energy can be saved in a battery for future use. Single-user channel capacity with an energy harvesting transmitter of an unlimited battery is equal to the capacity with an average power constraint equal to the average recharge rate [1], [2]. This result extends to the capacity region of the multiple access and broadcast channels with energy harvesting users having unlimited batteries [1], [2]. While the capacity with a finite battery is still an open problem, we found the capacity of an AWGN channel with an energy harvesting transmitter with no battery in [3] by using the relation of this problem with the problem of data transmission over statedependent channels. In this paper, we extend our work in [3] and address the capacity region of the Gaussian MAC with batteryless energy harvesting transmitters.

We consider two energy harvesting transmitters sending messages over an AWGN MAC as shown in Fig. 1. Exogenous energy sources supply $E_{1 i}$ and $E_{2 i}$ amounts of energies to users 1 and 2, respectively, at the $i$ th channel use and upon observing the arrived energy, users send a code symbol whose energy is constrained to the currently available energy. The channel input and output are related as

$$
Y_{i}=X_{1 i}+X_{2 i}+N_{i}, \quad i=1, \ldots, n
$$

This work was supported by NSF Grants CNS 09-64632, CCF 09-64645, CCF 10-18185 and CNS 11-47811.

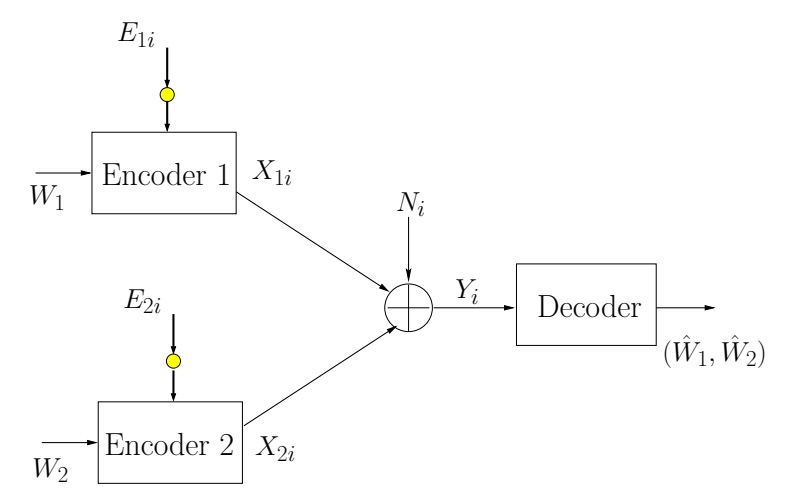

Fig. 1. The Gaussian MAC with batteryless energy harvesting transmitters.

where $X_{1 i}$ and $X_{2 i}$ are the channel inputs of users 1 and 2, respectively, and $Y_{i}$ is the channel output at the $i$ th channel use. $N_{i}$ is the i.i.d. Gaussian noise distributed as $\mathcal{N}(0,1)$. $E_{11}, \ldots, E_{1 n}$ and $E_{21}, \ldots, E_{2 n}$ are i.i.d. (in time) energy arrival sequences which are independent of the messages of the users. The code symbol energy at the $i$ th channel use is constrained according to the exogenous energy arrival. In particular, users 1 and 2 observe $E_{1 i}$ and $E_{2 i}$ and generate channel inputs $X_{1 i}$ and $X_{2 i}$ that satisfy $X_{1 i}^{2} \leq E_{1 i}$ and $X_{2 i}^{2} \leq E_{2 i}$, i.e., each code symbol is amplitude constrained to (the square root of) the observed energy. In [3], we addressed such a single-user system and found the single-user capacity by solving for the maximum mutual information between the input and output of an extended input channel [4].

In this paper, we extend our work in [3] to a MAC where the channel inputs are constrained to possibly correlated time-varying amplitude constraints. We first investigate the case of static amplitude constraints in the MAC setting. The literature on static amplitude constraints has generally covered the single-user case [5]-[9] for various channels including quadrature-amplitude constrained AWGN channel and Rayleigh and Ricean fading channels with and without state information. As a common result in [5]-[9], the optimal input distribution under amplitude constraint is discrete. Reference [10] considers a MAC with static amplitude constrains and shows that under small amplitude constraints, every point in the capacity region is achieved by binary input distributions. The recent independent and concurrent work [11] addresses the sum capacity of the Gaussian MAC with peak power 
constraints.

The variations in the available energy at the transmitter links the problem of data transmission with an energy harvesting transmitter to the problem of data transmission over statedependent channels: The energy level at the transmitter is a state that is available to only the transmitter. Single-user and multiple access state-dependent channels have been well investigated [12]-[17]. Specifically, when causal state information at the transmitters is available, Shannon strategies are capacity achieving for the single-user state-dependent channels and provide an achievable region for the state-dependent MAC [13]-[17].

In this paper, we first consider the Gaussian MAC with static amplitude constraints and show that the boundary of the capacity region is achieved by discrete input distributions of finite support. We, then, consider a MAC where transmitters are energy harvesting with no battery and provide an achievable region by Shannon strategies applied by each user. We show that the boundary of this achievable region is achieved by discrete input distributions with support set of zero Lebesgue measure in the corresponding Euclidean space. Finally, we provide numerical illustrations.

\section{Gaussian MAC with Static Amplitude CONSTRAINTS}

In this section, we consider the two-user Gaussian MAC with amplitude constrained inputs $\left|X_{1}\right| \leq A_{1}$ and $\left|X_{2}\right| \leq A_{2}$. The Gaussian MAC has the conditional density $p\left(y \mid x_{1}, x_{2}\right)=$ $\phi\left(y-x_{1}-x_{2}\right)$ where $x_{1}$ and $x_{2}$ are the channel inputs of users 1 and 2, respectively, $y$ is the channel output and $\phi(\tau)=\frac{1}{\sqrt{2 \pi}} e^{-\frac{\tau^{2}}{2}}$ is the zero-mean unit-variance Gaussian density. The feasible (i.e., amplitude constrained) marginal input distributions are given, respectively, as

$$
\begin{aligned}
& \Omega_{1}=\left\{F_{X_{1}}: \int_{-A_{1}}^{A_{1}} d F_{X_{1}}=1\right\} \\
& \Omega_{2}=\left\{F_{X_{2}}: \int_{-A_{2}}^{A_{2}} d F_{X_{2}}=1\right\}
\end{aligned}
$$

where $F_{X_{1}}$ and $F_{X_{2}}$ are the cumulative distribution functions. Given $F_{X_{1}}$ and $F_{X_{2}}$, the following region is achievable [18]:

$$
\begin{aligned}
R_{1} & \leq I\left(X_{1} ; Y \mid X_{2}\right) \\
R_{2} & \leq I\left(X_{2} ; Y \mid X_{1}\right) \\
R_{1}+R_{2} & \leq I\left(X_{1}, X_{2} ; Y\right)
\end{aligned}
$$

Note that the mutual information terms $I\left(X_{1}, X_{2} ; Y\right)$, $I\left(X_{1} ; Y \mid X_{2}\right)$ and $I\left(X_{2} ; Y \mid X_{1}\right)$ are functionals defined from $\Omega_{1} \times \Omega_{2}$ to $\mathbb{R}^{+} \cup\{0\}$. The capacity region of the MAC with input amplitude constraints is the convex hull of the union of the pentagons [18] in the form of (4)-(6).

Since the capacity region is convex [18], the pair of input distributions $\left(F_{X_{1}}, F_{X_{2}}\right)$ that achieves the boundary of the capacity region are found by solving optimization problems that are parametrized by the slope of the supporting hyperplanes (see Fig. 2). In particular, the sum-rate optimal pair of

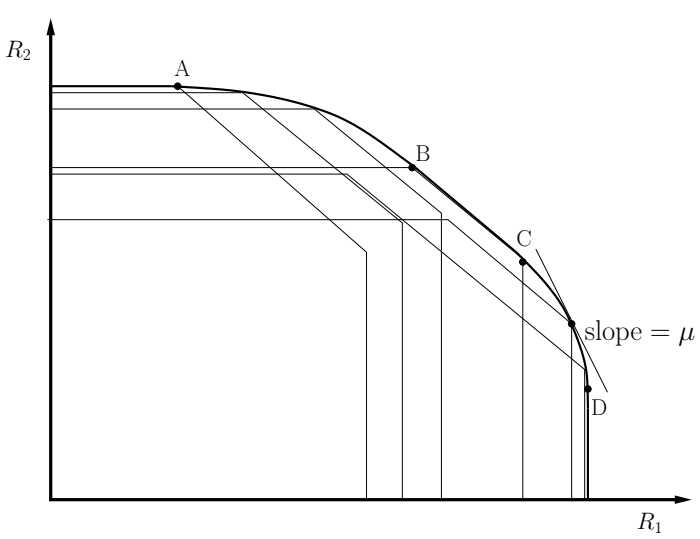

Fig. 2. The capacity region of Gaussian MAC with amplitude constraints.

distributions that achieves the time-sharing points between $B$ and $C$ in Fig. 2 is the solution of the following functional optimization problem:

$$
\max _{F_{X_{1}} \in \Omega_{1}, F_{X_{2}} \in \Omega_{2}} I\left(X_{1}, X_{2} ; Y\right)
$$

The boundary on the left of the sum-rate optimal points between $A$ and $B$ in Fig. 2 is achieved by a pair $\left(F_{X_{1}}, F_{X_{2}}\right)$ that is the solution of the following problem for some $\mu<1$

$$
\max _{F_{X_{1}} \in \Omega_{1}, F_{X_{2}} \in \Omega_{2}}(1-\mu) I\left(X_{2} ; Y \mid X_{1}\right)+\mu I\left(X_{1}, X_{2} ; Y\right)
$$

Similarly, the boundary on the right of the sum-rate optimal points between $C$ and $D$ in Fig. 2 is achieved by the solution of the following problem for some $\mu>1$

$$
\max _{F_{X_{1}} \in \Omega_{1}, F_{X_{2}} \in \Omega_{2}}(\mu-1) I\left(X_{1} ; Y \mid X_{2}\right)+I\left(X_{1}, X_{2} ; Y\right)
$$

In the sequel, we will focus on the solution of (9) since (7) is a special case of (9) for $\mu=1$ and the solution of (8) follows from symmetry. For convenience, we define the following:

$$
\begin{aligned}
& \widehat{Y}=X+N \\
& \widetilde{Y}=X_{1}+\widetilde{N} \\
& \bar{Y}=X_{2}+\bar{N}
\end{aligned}
$$

where $\tilde{N}=X_{2}+N$ for fixed $X_{2}$ and $\bar{N}=X_{1}+N$ for fixed $X_{1}$. $X$ in (10) can be either $X_{1}$ or $X_{2}$. We therefore note that $I\left(X_{1} ; Y \mid X_{2}\right)=I\left(X_{1} ; \widehat{Y}\right)$ and $I\left(X_{2} ; Y \mid X_{1}\right)=I\left(X_{2} ; \widehat{Y}\right)$. Moreover, $I\left(X_{1}, X_{2} ; Y\right)$ can be equivalently expressed as $I\left(X_{2} ; \bar{Y}\right)+I\left(X_{1} ; \widehat{Y}\right)$ and as $I\left(X_{1} ; \widetilde{Y}\right)+I\left(X_{2} ; \widehat{Y}\right)$.

We now provide several facts about the objective function and the feasible set in (9). The proofs of these facts follow from arguments similar to those in [8], [9] and therefore are skipped here for brevity. We first note that $\Omega_{1}$ and $\Omega_{2}$ are convex and sequentially compact function spaces. $I\left(X_{1}, X_{2} ; Y\right)$ is a continuous functional of the tuple $\left(F_{X_{1}}, F_{X_{2}}\right)$ on $\Omega_{1} \times \Omega_{2}$ and is strictly concave in $F_{X_{1}}$ given $F_{X_{2}}$ and vice versa. $I\left(X_{1} ; Y \mid X_{2}\right)=I\left(X_{1} ; \widehat{Y}\right)$ and $I\left(X_{2} ; Y \mid X_{1}\right)=I\left(X_{2} ; \widehat{Y}\right)$ are strictly concave functionals of only $F_{X_{1}}$ and only $F_{X_{2}}$, respectively. $I\left(F_{X_{1}}, F_{X_{2}}\right)$, an alternative notation for $I\left(X_{1}, X_{2} ; Y\right)$, 
is Frechet differentiable in both $F_{X_{1}}$ and $F_{X_{2}}$. We use the relation $I\left(X_{1}, X_{2} ; Y\right)=I\left(X_{2} ; \bar{Y}\right)+I\left(X_{1} ; \widehat{Y}\right)$. Given $F_{X_{1}}$, $I\left(X_{1} ; \widehat{Y}\right)$ is fixed and the derivative of $I\left(F_{X_{1}}, F_{X_{2}}\right)$ with respect to $F_{X_{2}}$ in the direction of $F_{X_{2}}^{\prime}$ is equal to the derivative of $I\left(X_{2} ; \bar{Y}\right)$, which is [5]:

$$
\begin{aligned}
& \lim _{\theta \rightarrow 0} \frac{1}{\theta}\left[I\left(F_{X_{1}}, \theta F_{X_{2}}^{\prime}+(1-\theta) F_{X_{2}}\right)-I\left(F_{X_{1}}, F_{X_{2}}\right)\right] \\
& =\int_{-A_{2}}^{A_{2}} h_{\bar{Y}}\left(x_{2} ; F_{X_{1}}, F_{X_{2}}\right) d F_{X_{2}}^{\prime}-h_{\bar{Y}}\left(F_{X_{1}}, F_{X_{2}}\right)
\end{aligned}
$$

where $h_{\bar{Y}}\left(x_{2} ; F_{X_{1}}, F_{X_{2}}\right)$ is the entropy density of $\bar{Y}$ generated by $F_{X_{1}}$ and $F_{X_{2}}$ :

$$
\begin{aligned}
& h_{\bar{Y}}\left(x_{2} ; F_{X_{1}}, F_{X_{2}}\right) \\
& \quad=-\int_{\mathbb{R}} p_{\bar{N}}\left(y-x_{2} ; F_{X_{1}}\right) \log \left(p_{\bar{Y}}\left(y ; F_{X_{1}}, F_{X_{2}}\right)\right) d y
\end{aligned}
$$

where $p_{\bar{N}}=\int_{-A_{1}}^{A_{1}} \phi\left(y-x_{1}\right) d F_{X_{1}}$ is the density of $\bar{N}$ given $F_{X_{1}}$ and $h_{\bar{Y}}\left(F_{X_{1}}, F_{X_{2}}\right)$ is the entropy of $p_{\bar{Y}}\left(y ; F_{X_{1}}, F_{X_{2}}\right)$. Similarly, we can express the derivative of $I\left(F_{X_{1}}, F_{X_{2}}\right)$ given $F_{X_{2}}$ with respect to $F_{X_{1}}$ in the direction of any other distribution in $\Omega_{1} . h_{\tilde{Y}}\left(x_{1} ; F_{X_{1}}, F_{X_{2}}\right)$ is defined similarly given $F_{X_{2}}$ :

$$
\begin{aligned}
& h_{\widetilde{Y}}\left(x_{1} ; F_{X_{1}}, F_{X_{2}}\right) \\
& \quad=-\int_{\mathbb{R}} p_{\widetilde{N}}\left(y-x_{1} ; F_{X_{2}}\right) \log \left(p_{\widetilde{Y}}\left(y ; F_{X_{1}}, F_{X_{2}}\right)\right) d y
\end{aligned}
$$

where $p_{\tilde{N}}=\int_{-A_{2}}^{A_{2}} \phi\left(y-x_{2}\right) d F_{X_{2}}$. Finally, we define

$$
h_{\widehat{Y}}\left(x ; F_{X}\right)=-\int_{\mathbb{R}} \phi(y-x) \log \left(p_{\widehat{Y}}\left(y ; F_{X}\right)\right) d y
$$

where $X$ can be either $X_{1}$ or $X_{2}$.

Note that in general the problem in (9) is not a convex optimization problem since the independence of $X_{1}$ and $X_{2}$ causes non-convexity. In particular, the objective function in (9) is not concave if it is viewed as a functional of tuples $\left(F_{X_{1}}, F_{X_{2}}\right)$. On the other hand, it is a strictly concave functional of the joint distribution of $\left(X_{1}, X_{2}\right)$ but the space of joint distributions generated by independent $X_{1}$ and $X_{2}$ marginal distributions is not a convex space. Therefore, finding the optimal $F_{X_{1}}$ and $F_{X_{2}}$ is challenging.

Note that since the objective function in (9) is strictly concave in marginal distributions, the solution of (9), denoted as $\left(F_{X_{1}}^{*}, F_{X_{2}}^{*}\right)$, necessarily satisfies the KKT optimality conditions. In particular, given $F_{X_{1}}^{*}$, the directional derivative of the objective function with respect to $F_{X_{2}}$ at $F_{X_{2}}^{*}$ in any direction must be less than or equal to zero with equality at $F_{X_{2}}^{*}$. Note that since $I\left(X_{1} ; Y \mid X_{2}\right)$ does not depend on $X_{2}$ for fixed $F_{X_{1}}$, the derivative of the objective function in (9) with respect to $F_{X_{2}}$ in the direction of $F_{X_{2}}^{\prime}$ is equal to the derivative in (13) and it should be less than or equal to zero for all $F_{X_{2}}^{\prime} \in \Omega_{2}$ :

$$
\int_{-A_{2}}^{A_{2}} h_{\bar{Y}}\left(x_{2} ; F_{X_{1}}^{*}, F_{X_{2}}^{*}\right) d F_{X_{2}}^{\prime} \leq h_{\bar{Y}}\left(F_{X_{1}}^{*}, F_{X_{2}}^{*}\right)
$$

One can show that (18) is equivalent to [5]:

$$
\begin{array}{ll}
h_{\bar{Y}}\left(x_{2} ; F_{X_{1}}^{*}, F_{X_{2}}^{*}\right) \leq h_{\bar{Y}}\left(F_{X_{1}}^{*}, F_{X_{2}}^{*}\right), & x_{2} \in\left[-A_{2}, A_{2}\right] \\
h_{\bar{Y}}\left(x_{2} ; F_{X_{1}}^{*}, F_{X_{2}}^{*}\right)=h_{\bar{Y}}\left(F_{X_{1}}^{*}, F_{X_{2}}^{*}\right), & x_{2} \in S_{F_{X_{2}}^{*}}
\end{array}
$$

where $S_{F_{X_{2}}^{*}}$ denotes the support set of $F_{X_{2}}^{*}$. Similarly, the corresponding condition for the directional derivative with respect to $F_{X_{1}}$ in the direction of $F_{X_{1}}^{\prime}$ given $F_{X_{2}}^{*}$ yields

$$
\begin{aligned}
\int_{-A_{1}}^{A_{1}} & {\left[(\mu-1) h_{\widehat{Y}}\left(x_{1} ; F_{X_{1}}^{*}\right)+h_{\widetilde{Y}}\left(x_{1} ; F_{X_{1}}^{*}, F_{X_{2}}^{*}\right)\right] d F_{X_{1}}^{\prime} } \\
& \leq(\mu-1) h_{\widehat{Y}}\left(F_{X_{1}}^{*}\right)+h_{\widetilde{Y}}\left(F_{X_{1}}^{*}, F_{X_{2}}^{*}\right)
\end{aligned}
$$

for all $F_{X_{1}}^{\prime} \in \Omega_{1}$ and we have the equivalent conditions

$$
\begin{aligned}
& (\mu-1) h_{\widehat{Y}}\left(x_{1} ; F_{X_{1}}^{*}\right)+h_{\widetilde{Y}}\left(x_{1} ; F_{X_{1}}^{*}, F_{X_{2}}^{*}\right) \\
& \quad \leq(\mu-1) h_{\widehat{Y}}\left(F_{X_{1}}^{*}\right)+h_{\widetilde{Y}}\left(F_{X_{1}}^{*}, F_{X_{2}}^{*}\right), \quad x_{1} \in\left[-A_{1}, A_{1}\right] \\
& (\mu-1) h_{\widehat{Y}}\left(x_{1} ; F_{X_{1}}^{*}\right)+h_{\widetilde{Y}}\left(x_{1} ; F_{X_{1}}^{*}, F_{X_{2}}^{*}\right) \\
& \quad=(\mu-1) h_{\widehat{Y}}\left(F_{X_{1}}^{*}\right)+h_{\widetilde{Y}}\left(F_{X_{1}}^{*}, F_{X_{2}}^{*}\right), \quad x_{1} \in S_{F_{X_{1}}^{*}}
\end{aligned}
$$

Note that for given $F_{X_{1}}^{*}, I\left(X_{1} ; Y \mid X_{2}\right)$ does not depend on $F_{X_{2}}$; however, for given $F_{X_{2}}^{*}$, both terms in the objective function (9) depend on $F_{X_{1}}$.

Next, we show that the necessary optimality conditions in (19)-(20) and (22)-(23) imply that the solution of (9), which is guaranteed to exist due to the continuity of the objective function and the compactness of the input distribution space, must be a discrete distribution. We first show that the conditions in (19)-(20) imply that $F_{X_{2}}^{*}$ is discrete. Note that given $F_{X_{1}}^{*}$, (19)-(20) are optimality conditions for finding the capacity of the single-user channel between $X_{2}$ and $\bar{Y}=X_{2}+\bar{N}$. We claim that for any $F_{X_{1}}^{*} \in \Omega_{1}$, $p_{\bar{N}}(y)=\int \phi\left(y-x_{1}\right) d F_{X_{1}}^{*}$ is in the class of noise densities in [8] for which the optimal input distribution is discrete under an amplitude constraint. Specifically, we verify the conditions i-iv in [8]: $p_{\bar{N}}(y)>0$ for all $y \in \mathbb{R}$ and $E\left[|Z|^{2}\right]<\infty$. Moreover, $p_{\bar{N}}(z)=\int \phi\left(z-x_{1}\right) d F_{X_{1}}^{*}$ is analytic over the whole complex plane $\mathbb{C}$. It suffices to use the analyticity of $p_{\bar{N}}(z)$ over the region $|\Im(z)|<\delta$ for some $\delta>0$. We next define:

$$
\begin{aligned}
& L(|\Re(z)|) \triangleq \frac{1}{\sqrt{2 \pi}} e^{-\frac{1}{2}\left(|\Re(z)|^{2}+A_{2}^{2}+2 A_{2}|\Re(z)|\right)} \\
& U(|\Re(z)|) \triangleq \frac{1}{\sqrt{2 \pi}} e^{-\frac{1}{2}\left((|\Re(z)|)^{2}-2 A_{2}|\Re(z)|-\delta^{2}\right)}
\end{aligned}
$$

One can show that $0<L(|\Re(z)|) \leq\left|p_{Z}(z)\right| \leq U(|\Re(z)|)$ for all $z \in \mathbb{C}$ with $|\Im(z)|<\delta$ and $|\Re(z)|>k$ where $k$ is sufficiently large. Moreover, for this selected $k$, $-\int_{k}^{\infty} U(\tau) \log (U(\tau)) d \tau<\infty$ and $\int_{x+k}^{\infty} \frac{U^{3}(\tau-x)}{L^{2}(\tau)} d \tau$ for all $x \in \mathbb{R}$. This proves that the support set of $F_{X_{2}}^{*}$ is a discrete set for any given arbitrary distribution $F_{X_{1}}^{*}$ in $\Omega_{1}$.

Now, we prove that conditions in (22)-(23) imply that $F_{X_{1}}^{*}$ is discrete given $F_{X_{2}}^{*}$ in $\Omega_{2}$. To this end, we assume $S_{F_{X_{1}}^{*}}$ is infinite and reach a contradiction. By BolzanoWeirestrass Theorem, $S_{F_{X_{1}}^{*}}$ has an accumulation point. 
Note that $\int \phi\left(y-x_{1}\right) \log \left(p_{Y \mid X_{2}}\left(y ; F_{X_{1}}^{*}\right)\right) d y$ and $\int p_{\widetilde{N}}(y-$ $\left.x_{1}\right) \log \left(p_{\tilde{Y}}\left(y ; F_{X_{1}}^{*}, F_{X_{2}}^{*}\right)\right) d y$ are analytic functions of $x_{1}$ and they have extension over the whole complex plane $\mathbb{C}$. By identity theorem of complex analysis and the optimality condition in (21), we have $\forall x_{1} \in \mathbb{C}$ and in particular $\forall x_{1} \in \mathbb{R}$ :

$$
\begin{aligned}
(\mu-1) & \int_{\mathbb{R}} \phi\left(y-x_{1}\right) \log \left(p_{\widehat{Y}}\left(y ; F_{X_{1}}^{*}\right)\right) d y+ \\
& \int_{\mathbb{R}} p_{\widetilde{N}}\left(y-x_{1}\right) \log \left(p_{\widetilde{Y}}\left(y ; F_{X_{1}}^{*}, F_{X_{2}}^{*}\right)\right) d y=D
\end{aligned}
$$

where $D=-(\mu-1) h_{\widehat{Y}}\left(F_{X_{1}}^{*}\right)-h_{\widetilde{Y}}\left(F_{X_{1}}^{*}, F_{X_{2}}^{*}\right)$. However, (26) causes a contradiction. Note that $p_{\widehat{Y}}\left(y ; F_{X_{1}}^{*}\right)$ is a well defined density function and hence $\log \left(p_{\widehat{Y}}\left(y ; F_{X_{1}}^{*}\right)\right) \rightarrow-\infty$ as $y \rightarrow \infty$. Consequently, $\int_{\mathbb{R}} \phi\left(y-x_{1}\right) \log \left(p_{\widehat{Y}}\left(y ; F_{X_{1}}^{*}\right)\right) d y$ also diverges to $-\infty$ as $x_{1}$ gets large since the window of $\phi\left(y-x_{1}\right)$ integrates over large $y$ values if $x_{1}$ is selected sufficiently large. Since $p_{\widetilde{N}}(y)=\int_{-A_{2}}^{A_{2}} \phi\left(y-x_{2}\right) d F_{X_{2}}$ shows the same windowing property as the Gaussian pdf $\phi($.$) in$ view of the fact that $A_{2}$ is finite, we have $\int_{\mathbb{R}} p_{\widetilde{N}}(y-$ $\left.x_{1}\right) \log \left(p_{\tilde{Y}}\left(y ; F_{X_{1}}^{*}, F_{X_{2}}^{*}\right)\right) d y \rightarrow-\infty$ as $x_{1} \rightarrow \infty$. This contradicts (26). Therefore, we have the following theorem:

Theorem $1 S_{F_{X_{1}}^{*}}$ and $S_{F_{X_{2}}^{*}}$ are finite sets.

Theorem 1 states that rate tuples on the boundary of the capacity region of the Gaussian MAC with amplitude constraints is achieved by discrete input distributions of finite support. In [10, Proposition 3], Verdú observed that if the output distributions $p_{Y}, p_{Y \mid X_{1}}$ and $p_{Y \mid X_{2}}$ are all unimodal, which holds if amplitude constraints are sufficiently small, then the capacity region is the pentagon generated by independent equiprobable binary input distributions located at $\pm A_{1}$ and $\pm A_{2}$. Recently, independent and concurrent work in [11] showed that the sum capacity of the Gaussian MAC is achieved by discrete distributions. Theorem 1 generalizes Smith's result for a single-user AWGN channel [5] to aa AWGN MAC, and the results in [10], [11] to the entire region.

\section{GAussian MAC With BATteryless ENERGY HARVESTING TRANSMITTERS}

In this section, we consider the Gaussian MAC where the energy required for data transmission is maintained by an exogenous joint energy arrival process and users have no battery to save energy. For convenience, we consider only two users and assume that the energy harvesting processes at both users take binary values $\mathcal{E}_{1}=\left\{e_{11}, e_{12}\right\}$ and $\mathcal{E}_{2}=\left\{e_{21}, e_{22}\right\}$. However, our analysis can be generalized for any finite value of $\left|\mathcal{E}_{1}\right|$ and $\left|\mathcal{E}_{2}\right|$. The joint energy arrival process is i.i.d. in time with $P\left(E_{1 i}=e_{1 k}, E_{2 i}=e_{2 l}\right)=p_{k l}$ for all $i$ where $\sum_{k, l} p_{k l}=1 . p_{1}=\sum_{l} p_{1 l}$ is the marginal probability that $e_{11}$ arrives at user 1 and $p_{2}=\sum_{k} p_{k 1}$ is the marginal probability that $e_{21}$ arrives at user 2 .

The amplitude constraints on $x_{1}$ and $x_{2}$ are time-varying according to the energy arrival process. Users 1 and 2 have messages $w_{1} \in \mathcal{W}_{1}$ and $w_{2} \in \mathcal{W}_{2}$, respectively. As the energies available for users at each channel use vary as an i.i.d. process and is independent of the messages of the users $w_{1}, w_{2}$, the resulting channel is an instance of a statedependent MAC with causal state information at the users [3], [4], [14] where the state is the available energy of users. In particular, we can associate four different states $(k, l)$, $k, l=1,2$ where at each state $(k, l)$, we have $\left|X_{1}\right| \leq \sqrt{e_{1 k}}$ and $\left|X_{2}\right| \leq \sqrt{e_{2 l}}$.

Capacity region of state-dependent MAC is still unknown; however, Shannon strategies provide an achievable region. In particular, let the state information at the users be $S_{U_{1}}$ and $S_{U_{2}}$, respectively, which are in general dependent. Let deterministic functions of $S_{U_{1}}$ and $S_{U_{2}}$ be $T_{1}=f_{1}\left(S_{U_{1}}\right)$ and $T_{2}=f_{2}\left(S_{U_{2}}\right)$. Then, the following rate region is achievable:

$$
\begin{aligned}
R_{1} & \leq I\left(T_{1} ; Y \mid T_{2}\right) \\
R_{2} & \leq I\left(T_{2} ; Y \mid T_{1}\right) \\
R_{1}+R_{2} & \leq I\left(T_{1}, T_{2} ; Y\right)
\end{aligned}
$$

Achievability of the region in (27)-(29) follows from [14, Section IV]. Note that the state of the channel in the energy harvesting MAC problem has two components (the energy arrivals at the two users) as in [16] and only one or both components of the state may be available to the users. In the following, we study achievable rate regions using Shannon strategies under the availability of one or both of the components of the energy state to the users.

\section{A. Joint Energy Arrival Information Available at Both Users}

When the state information $\left(e_{1 k}, e_{2 l}\right)$ is available to both users perfectly, full state information of the multiple access channel is available at the users. Let $T_{k l}^{(1)}$ and $T_{k l}^{(2)}$ denote the code symbols generated by users 1 and 2, respectively, upon observing that the joint energy arrival $\left(E_{1}, E_{2}\right)=\left(e_{1 k}, e_{2 l}\right)$, $k, l=1,2$, occurred. The conditional density of the extended MAC with inputs $T_{k l}^{(1)}$ and $T_{k l}^{(2)}$ and output $Y$ is:

$$
p\left(y \mid t_{k l}^{(1)}, t_{k l}^{(2)}\right)=\sum_{k l} p_{k l} \phi\left(y-t_{k l}^{(1)}-t_{k l}^{(2)}\right)
$$

Note that $\left|T_{k l}^{(1)}\right| \leq \sqrt{e_{1 k}}$ and $\left|T_{k l}^{(2)}\right| \leq \sqrt{e_{2 l}}$. For $k, l=1,2$, $\left\{T_{k l}^{(1)}\right\}$ and $\left\{T_{k l}^{(2)}\right\}$ are jointly distributed and $\left\{T_{k l}^{(1)}\right\}$ are independent of $\left\{T_{k l}^{(2)}\right\}$. The region in (27)-(29) evaluated for $T_{1}=T_{k l}^{(1)}$ and $T_{2}=T_{k l}^{(2)}$ is achievable. Achievability of this region also follows from [15, Theorem 3]. Moreover, [15, Theorem 3] provides an outer bound for the capacity region by allowing cooperation between the users.

\section{B. Each User Has Its Own Energy Arrival Information}

Now, we consider the scenario in which user 1 does not know the energy arrival of user 2 and vice versa. This scenario can be viewed as a state-dependent MAC with partial state information at the transmitter as in [14] or with only a component of the state available to each user as in [16]. However, note that the components of the states may not be independent unlike [16]. Let $T_{k}^{(1)}$ and $T_{l}^{(2)}$ denote the code symbols generated by users 1 and 2, respectively, upon user 1's observation that $E_{1}=e_{1 k}, k=1,2$, occurred and user 
2's observation that $E_{2}=e_{2 l}, l=1,2$ occurred. The resulting extended input alphabet with inputs $T_{k}^{(1)}$ and $T_{l}^{(2)}$ and output $Y$ has the following conditional density

$$
p\left(y \mid t_{k}^{(1)}, t_{l}^{(2)}\right)=\sum_{k l} p_{k l} \phi\left(y-t_{k}^{(1)}-t_{l}^{(2)}\right)
$$

where $\left|T_{k}^{(1)}\right| \leq \sqrt{e_{1 k}}, k=1,2$ and $\left|T_{l}^{(2)}\right| \leq \sqrt{e_{2 l}}, l=1,2$. $T_{1}^{(1)}, T_{2}^{(1)}$ are jointly distributed and they are independent of the other jointly distributed pair $T_{1}^{(2)}$ and $T_{2}^{(2)}$. The rate region evaluated at $T_{1}=T_{k}^{(1)}$ and $T_{2}=T_{l}^{(2)}$ in (27)-(29) is achievable. We note that if energy arrivals of the users $E_{1}$ and $E_{2}$ are independent, then the users have independent channel state information and the sum-rate yielded by Shannon strategies is the sum-rate capacity from [14, Theorem 4].

In both cases, the boundary of the achievable region is found by solving optimization problems as in (7)-(9) by replacing the sum rate and individual rate constraints accordingly. By using similar steps, we can establish that the capacity achieving input distributions have zero Lebesgue measure in $\mathbb{R}^{2}$. We next sketch this extension. We have the following problem:

$$
\begin{aligned}
\max & (\mu-1) I\left(T_{1}^{(1)}, T_{2}^{(1)} ; Y \mid T_{1}^{(2)}, T_{2}^{(2)}\right) \\
& +I\left(T_{1}^{(1)}, T_{2}^{(1)}, T_{1}^{(2)}, T_{2}^{(2)} ; Y\right) \\
\text { s.t. } & F_{T_{1}^{(1)}, T_{2}^{(1)}} \in \Theta_{1}, \quad F_{T_{1}^{(2)}, T_{2}^{(2)}} \in \Theta_{2}
\end{aligned}
$$

where

$$
\begin{aligned}
& \Theta_{1}=\left\{F_{T_{1}^{(1)}, T_{2}^{(1)}}: \int_{-\sqrt{e_{11}}}^{\sqrt{e_{11}}} \int_{-\sqrt{e_{12}}}^{\sqrt{e_{12}}} d F_{T_{1}^{(1)}, T_{2}^{(1)}}=1\right\} \\
& \Theta_{2}=\left\{F_{T_{1}^{(2)}, T_{2}^{(2)}}: \int_{-\sqrt{e_{21}}}^{\sqrt{e_{21}}} \int_{-\sqrt{e_{22}}}^{\sqrt{e_{22}}} d F_{T_{1}^{(2)}, T_{2}^{(2)}}=1\right\}
\end{aligned}
$$

are the feasible sets of joint distributions. As in the static amplitude constrained case, $\Theta_{1}$ and $\Theta_{2}$ are convex and sequentially compact spaces. Moreover, the objective function in (32) is Frechet differentiable and strictly concave in $F_{T_{1}^{(1)}, T_{2}^{(1)}}$ and $F_{T_{1}^{(2)}, T_{2}^{(2)}}$, separately. Next, we use the KKT optimality conditions for $F_{T_{1}^{(2)}, T_{2}^{(2)}}^{*}$ given $F_{T_{1}^{(1)}, T_{2}^{(1)}}^{*}$ in terms of the mutual information densities, which hold with equality at the support set of the optimal input distributions. Assuming that the support set has nonzero Lebesgue measure and using analyticity of the mutual information densities in two-dimensional complex plane, we conclude that the optimality condition holds with equality for the entire two-dimensional real plane but it causes a contradiction along the $t_{1}=t_{2}$ line as in [3]. This proves that $F_{T_{1}^{(2)}, T_{2}^{(2)}}^{*}$ has zero Lebesgue measure in $\mathbb{R}^{2}$ given $F_{T_{1}^{(1)}, T_{2}^{(1)}}^{(1)}$. We repeat this procedure to prove the same for $F_{T_{1}^{(1)}, T_{2}^{(1)}}^{*}$ given $F_{T_{1}^{(2)}, T_{2}^{(2)}}^{*}$.

Theorem $2 S_{F_{T_{1}^{*}}^{(1)}, T_{2}^{(1)}}$ and $S_{F_{T_{1}^{*}, T_{2}^{*}}^{(2)}}$ have zero Lebesgue measure in $\mathbb{R}^{2}$.

For both of the possible available information cases, the general shape of the achievable rate region is as in Fig. 2. At points $D$ and $A$, users 1 and 2, respectively, achieve maximum single-user rates with Shannon strategies $C_{S h}^{(1)}$ and $C_{S h}^{(2)}$. To illustrate, $C_{S h}^{(1)}$ is the maximum mutual information between the input and output of the following extended input channel:

$$
p\left(y \mid t_{1}, t_{2}\right)=p_{1} \phi\left(y-t_{1}\right)+\left(1-p_{1}\right) \phi\left(y-t_{2}\right)
$$

where $\left|t_{1}\right| \leq \sqrt{e_{11}},\left|t_{2}\right| \leq \sqrt{e_{12}}$. $p_{1}$ is the marginal probability that $e_{11}$ arrives. We note that $C_{S h}^{(1)}$ or $C_{S h}^{(2)}$ can always be achieved by letting $X_{2}=0$ or $X_{1}=0$ for any energy arrival, i.e., by creating no interference for the other user.

Note that in the MAC setting, individual users may achieve higher rates than $C_{S h}^{(1)}$ or $C_{S h}^{(2)}$. The potential boost in singleuser rates can be provided by the other user's help: If the energy arrivals of the users are correlated or one user knows the other user's energy state information, then that user may convey the energy state information to the receiver using block Markov encoding [16] and the receiver then decodes the other user's message given this state information. This way, a user may better help the other one than just creating no interference.

\section{Numerical Results}

In this section, we numerically study the optimal input distributions and the resulting capacity or achievable regions.

\section{A. Static Amplitude Constraints}

First, we focus on small amplitude constraints. We numerically observe that for the unit noise variance, if $A_{1} \leq 1.3$ and $A_{2} \leq 1.3$, the unimodality condition in [10, Proposition 3] holds and binary input distributions are optimal. We numerically verify ${ }^{1}$ that indeed binary distributions are optimal for $A_{1} \leq 1.6$ and $A_{2} \leq 1.6$.

We let $A_{1}=1.3$ and $A_{2}=2$. The single-user capacity under $A_{1}=1.3$ is achieved by symmetric binary distribution at \pm 1.3 and the single-user capacity under $A_{2}=2$ is achieved by ternary distribution located at 0 and \pm 2 . We observe in our numerical study ${ }^{2}$ that the optimal input distribution for user 1 is always binary for any $\mu \geq 0$ and this enables us to determine the capacity region for this particular case. However, the optimal input distribution for user 2 varies: for $\mu=1$, i.e., for the maximum sum-rate, binary input distribution is optimal. For some $\mu<1$, ternary input distribution is optimal.

We plot the resulting capacity region with $A_{1}=1.3$ and $A_{2}=2$ in Fig. 3 and compare it with the capacity region with $A_{1}=1.3$ and $A_{2}=1.6$. We observe that the latter capacity region is a pentagon and the optimal distributions are binary for both users. When the amplitude constraint of user 2 is increased, the capacity region becomes curved.

\section{B. On-Off Energy Arrivals}

In this section, we consider binary on-off energy arrivals with $e_{11}=0, e_{12}=1, e_{21}=0$ and $e_{22}=2.25$. $p_{k l}=0.25$ for all $k, l=1,2$, i.e., the energy arrivals of the

${ }^{1}$ We numerically verify the necessary optimality conditions in (19)-(20) and (22)-(23) for the binary distribution.

${ }^{2}$ By numerically studying (19)-(20) and (22)-(23), we observe that for any $X_{2}$ distribution, binary distribution on $X_{1}$ maximizes $I\left(X_{1} ; X_{1}+X_{2}+N\right)$. 


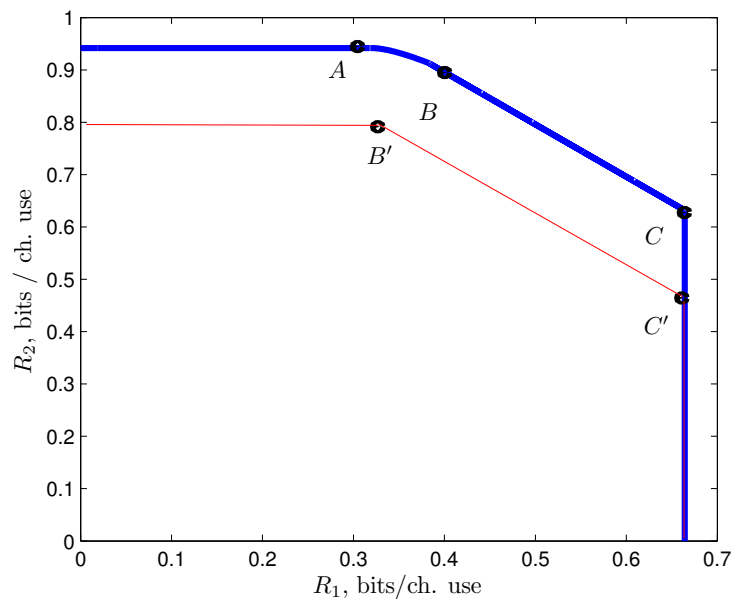

Fig. 3. The capacity regions of Gaussian MAC under amplitude constraints $A_{1}=1.3, A_{2}=1.6$ (the smaller region) and $A_{1}=1.3$ and $A_{2}=2$ (the larger region).

users are independent. We plot in Fig. 4 (the smaller region) the achievable rate region under only individual energy state information. We observe that the single-user rates $C_{S h}^{(1)}$ and $C_{S h}^{(2)}$ are achievable only if the other user's rate is zero. We also observe that the optimal sum-rate is achieved by binary input distributions ${ }^{3}$. Note that since the energy arrivals of the users are independent, by [14, Theorem 4] the sum-rate capacity is the optimal sum-rate achieved by Shannon strategies. Next, we plot in Fig. 4 (the larger region) the capacity region when energy state information is available to the transmitters and the receiver, which is an outer bound for the case of state information at only the transmitters. Note that this region is obtained by averaging the regions constrained by amplitude constraints due to each energy arrival over the joint energy arrival process. There is a large gap between the achievable region and the outer bound. This is partly because the naive Shannon strategy does not take advantage of block Markov encoding [16]. However, there is strong evidence from [3] that the achievable rates under energy state information at only the transmitters may be significantly lower than those under energy state information at both sides. We also observe in our numerical study that the cooperative outer bound in $[15$, Theorem 3] yields a looser outer bound.

\section{Conclusions}

We studied the Gaussian MAC with batteryless energy harvesting transmitters. We first considered MAC with static amplitude constraints and proved that the boundary of the capacity region is achieved by input distributions of finite support. Next, we considered discrete time-varying amplitude constraints. We provided achievable regions by Shannon strategies and proved that the boundary of these regions is achieved by input distributions with support set of zero Lebesgue measure in the corresponding Euclidean space.

\footnotetext{
${ }^{3}$ Although Theorem 2 does not imply it, this numerical study shows that the optimal input distributions for the optimization problem in (32) may have finite support set.
}

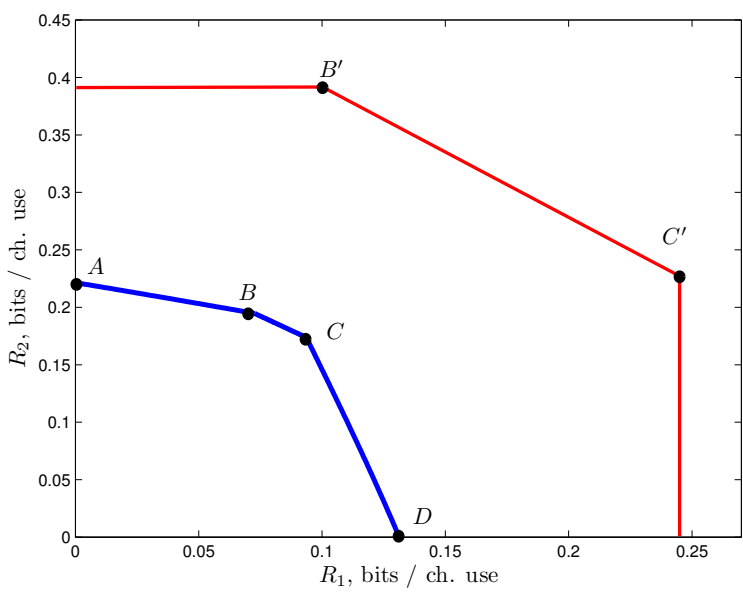

Fig. 4. The achievable region (the smaller region) and an outer bound (the larger region) for the Gaussian MAC under on-off energy arrivals with causal individual energy state information at the users.

\section{REFERENCES}

[1] O. Ozel and S. Ulukus, "Information theoretic analysis of an energy harvesting communication system," in Workshop on Green Wireless (WGREEN) at IEEE PIMRC, September 2010.

[2] O. Ozel and S. Ulukus, "Achieving AWGN capacity under stochastic energy harvesting," IEEE Trans. on Inform. Theory, to appear.

[3] O. Ozel and S. Ulukus, "AWGN channel under time-varying amplitude constraints with causal information at the transmitter," in Asilomar Conference on Signals, Systems and Computers, November 2011.

[4] C. Shannon, "Channels with side information at the transmitter," IBM Jour. of Research and Development, vol. 2, October 1958.

[5] J. G. Smith, "The information capacity of amplitude and varianceconstrained scalar Gaussian channels," Information and Control, vol. 18 , pp. 203-219, April 1971.

[6] I. Abu-Faycal, M. Trott, and S. Shamai, "The capacity of discretetime memoryless rayleigh fading channels," IEEE Trans. on Information Theory, vol. 47, pp. 1290-1301, May 2001.

[7] M. C. Gursoy, H. V. Poor, and S. Verdu, "The noncoherent ricean fading channel part-I: Structure of the capacity achieving input," IEEE Trans. Wireless Commun., vol. 4, pp. 2193-2206, September 2005.

[8] A. Tchamkerten, "On the discreteness of capacity achieving distributions," IEEE Trans. on Inform. Theory, vol. 50, pp. 2273-2278, November 2004.

[9] T. H. Chan, S. Hranilovic, and F. Kschischang, "Capacity-achieving probability measure for conditionally Gaussian channels with bounded inputs," IEEE Trans. Inform. Theory, vol. 51, pp. 2073-2088, June 2005.

[10] S. Verdu, "Capacity region of Gaussian CDMA channels: The symbolsynchronous case," in Allerton Conference, October 1986.

[11] B. Mamandipoor, K. Moshksar, and A. Khandani, "On the sum-capacity of Gaussian MAC with peak constraint," in IEEE ISIT, July 2012.

[12] G. Caire and S. Shamai, "On the capacity of some channels with channel state information," IEEE Trans. on Inform. Theory, vol. 45, pp. 20072019, September 1999.

[13] G. Keshet, Y. Steinberg, and N. Mehrav, "Channel coding in the presence of side information," Foundations and Trends in Communications and Information Theory, vol. 4, no. 6, pp. 445-586, 2007.

[14] S. A. Jafar, "Capacity with causal and non-causal side information- a unified view," IEEE Trans. on Inform. Theory, vol. 52, pp. 5468-5474, December 2006

[15] S. Sigurjonsson and Y.-H. Kim, "On multiple user channels with state information at the transmitters," in IEEE ISIT, September 2005.

[16] A. Lapidoth and Y. Steinberg, "The multiple access channel with two independent states each known causally to one encoder," in IEEE ISIT, June 2010.

[17] M. Li, O. Simeone, and A. Yener, "Multiple access channels with states causally known at the transmitters," IEEE Trans. on Inform. Theory, submitted, November 2010. Also available at ArXiv:[1011.6639].

[18] T. M. Cover and J. Thomas, Elements of Information Theory. John Wiley and Sons Inc., 2006. 